\title{
Pre-Discharge Screening Trans-Cutaneous Bilirubinometry in Healthy Newborns in Mahdieh Hospital, Tehran
}

\author{
Abolfazl Afjeh ${ }^{1,2} ;$ Minoo Fallahi $^{1,2,} ;$ Mehrnoosh Jahanbeen $^{3}$; Azita Basiri $^{2} ;$ Mastaneh Allaee $^{4}$ \\ ${ }^{1}$ Neonatal Health Research Center (NHRC), Mofid Children's Hospital, Tehran, IR Iran \\ ${ }^{2}$ Mahdieh Hospital, Tehran, IR Iran \\ ${ }^{3}$ Shohada Hospital, Shahid Beheshti University of Medical Sciences, Tehran, IR Iran \\ 4 Blood Transfusion Research Center, High Institute for Research and Education in Transfusion, Tehran, IR Iran \\ *Corresponding author: Minoo Fallahi, Neonatal Health Research Center (NHRC), Mofid Children's Hospital, Tehran, IR Iran. E-mail: minoofallahi@yahoo.com
}

Received: May 5, 2014; Accepted: April 15, 2015

\begin{abstract}
Background: Incidence of jaundice is high in newborn infants. Since well appearing newborns are rapidly and routinely discharged from hospital, performing an inexpensive noninvasive pre-discharge screening test for evaluation of jaundice seems to be necessary. Objectives: This study was conducted to compare the accuracy of cutaneous v/s serum bilirubin measurements in this regard.

Patients and Methods: This was a prospective cross sectional study conducted in Mahdieh hospital, Tehran. 613 neonates weighing $\geq$ $1,800 \mathrm{~g}$ with gestational age of $\geq 35$ weeks were enrolled. A pre discharge transcutaneous bilirubin test(TcB) was performed in all. Serum samples were taken from neonates with $\mathrm{TcB} \geq 5 \mathrm{mg} / \mathrm{dL}$ in first and $>8 \mathrm{mg} / \mathrm{dL}$ in second 24 hours. Decision for treatment or recheck of bilirubin level after discharge was made based on serum bilirubin results.

Results: Based on the study protocol, among 613 studied neonates, 491 (80\%) revealed high TcB, of them 240 (49\%) cases showed TBC $\geq 5$ $\mathrm{mg} / \mathrm{dL}$ in first and 251 (51\%) in second pre-discharge 24 hours. TcB ranged 3.3 - 17.1, mean TcB in first 24 hours was $6.9 \pm 1.7$ (mode 6) and in second 24 hours $9.1 \pm 2.1$ (mode 10). Of 491 neonates with high TcB, capillary serum sample was taken as the second step and 398 neonates revealed high total serum bilirubin (TsB) with the same protocol for TcB. 108 (27.1\%) neonates showed TsB $\geq 5 \mathrm{mg} / \mathrm{dL}$ in first and 290 (72.9\%) in second 24 hours. According to the study results TcB has a 81\% positive predictive value (PPV) in diagnosis of hyperbilirubinemia. Correlation coefficient of TcB and TsB in highest rate is equal to $72 \%$ ( $P$ value $<0.001$ ).

Conclusions: TcB is an inexpensive, noninvasive and precise pre-discharge screening test for evaluation of hyperbilirubinemia, with a high PPV. It is highly recommended to be performed routinely due to high incidence of hyperbilirubinemia in neonates.
\end{abstract}

Keywords: Bilirubin; Hyperbilirubinemia; Transcutaneous; Predischarge Screening; TcB

\section{Background}

Jaundice is one of the most common diseases in neonatal period. More than $60 \%$ of newborns develop clinical jaundice in the first week of life $(1,2)$. Pathologic levels of bilirubin may lead to irreversible complications such as bilirubin encephalopathy called kernicterus associated with cerebral palsy, deafness, dental dysplasia, ophthalmoplasia with upward gaze, and personality disorders (3, 4). Early discharge of neonates from nursery aggravates its danger (5). Awareness of risk factors causing severe jaundice, and early diagnostic and treatment interventions may prevent this severe irreversible complication. Estimation of the severity of jaundice and anticipation of its progression solely based on clinical observations is often not accurate enough and could lead to missed diagnosis. Transcutaneous bilirubin (TcB) measurement may be an effective method for prompt diagnosis of jaundice and prevention of its rapid progression (3). Some studies have recommended predischarge bilirubin screening for early detection of neonates at risk, so that treatment could be started as soon as possible, although evidences showing better outcome for these neonates is inadequate (6).

According to the American academy of pediatrics guidelines, all neonates should be evaluated for hyperbilirubinemia before discharge from hospital and serum bilirubin should be checked in neonates at risk (7). Moreover, the Canadian Academy of Pediatrics recommends serum bilirubin levels measurement by TcB (transcutaneous bilirubin) and TsB (total serum bilirubin) 24 to 72 hours after birth (prior to hospital discharge) for all neonates (8). TcB may be used as an estimate of the bilirubin level, since in various studies linear relationship between TcB and TsB has been specified ( $r=0.87$ to 0.96 ).

Although hyperbilirubinemia is highly prevalent in Iran and neonates are usually discharged before 24 hours of delivery, no strict evaluation for hyperbilirubinemia currently must be done.

\section{Objectives}

The goal of our study was comparison of transcutane-

Copyright (C) 2015, Growth \& Development Research Center. This is an open-access article distributed under the terms of the Creative Commons Attribution-NonCommercial 4.0 International License (http://creativecommons.org/licenses/by-nc/4.0/) which permits copy and redistribute the material just in noncommercial usages, provided the original work is properly cited. 
ous and serum bilirubin levels in order to determine the sensitivity (positive predictive value) of this non invasive method in term and late preterm infants.

\section{Patients and Methods}

This cross-sectional prospective study was conducted between 22 December 2010 and 20 March 2011 in Mahdieh hospital, a maternity university hospital in Tehran, Iran covered by Shahid Beheshti university and has 4,000 - 5,000 annually delivery. The study had received the approval of ethics committee of Shahid Beheshti university. Inclusion criterion was all healthy term and some late preterm neonates (those with gestational age more than 35 weeks) with birth weight over $1,800 \mathrm{~g}$ that were born in this period of time. In addition to physical examination for visible jaundice, the neonates were tested by forehead billi check 8,000 KJ Kejian, transcutaneous bilirubinometery just before discharge from hospital. In neonates with TcB levels of bilirubin $\geq 5$ $\mathrm{mg} / \mathrm{dL}$ in 24 hours after birth and $\geq 8 \mathrm{mg} / \mathrm{dL}$ in age of 48 hours, serum bilirubin level was checked by drawing capillary blood from foot heel to compare the results with TcB.

Selection of the above mentioned cut off levels was based on minimal pathologic level of serum bilirubin according to age specific Bhutani curve in American acad- emy of pediatrics (AAP) guideline in neonatal hyperbilirubinemia (7). In cases with high serum bilirubin levels, neonates were admitted to neonatal wards for phototherapy, but in cases with lower bilirubin levels neonates were discharged from hospital advising parents to come back at a specific time interval for rechecking bilirubin to excise risk factors.

Our exclusion criteria were neonates with birth weight lower than 1,800 g and gestational age less than 35 weeks, as well as sick babies who needed admission for other causes in special care units.

All demographic data including mother's and infant's blood group, neonate's gestational age, sex, and birth weight, mode of delivery, and bilirubin levels obtained by both TcB and TsB methods were registered in designed check lists. Data were analyzed by SPSS software.

\section{Results}

During the study period, 800 newborns were delivered in Mahdieh hospital and 613 neonates were enrolled in study. Of these $51 \%$ were boys, $57.5 \%$ were delivered by cesarean section. Mean birth weight was 3130 gram and mean gestational age 38.2 weeks.

TcB was performed in all cases just before discharge from hospital. Demographic characteristics of patients are shown in Table 1.

\begin{tabular}{|c|c|c|c|c|c|c|}
\hline Characteristics & $\mathrm{TcB}+, \%^{\mathrm{b}}$ & Total Numbers & PValue & TsB,$+ \%^{c}$ & TsB-, \% (n) & PValue \\
\hline Sex & & & 0.22 & & & 0.2 \\
\hline Male & $250(50.9)$ & $306(49.9)$ & & $201(50.5)$ & $49(52.6)$ & \\
\hline Female & $241(49.1)$ & $307(50.1)$ & & $197(49.5)$ & $44(47.3)$ & \\
\hline Weight, g & & & 0.034 & & & 0.09 \\
\hline$<2500$ & $27(14.1)$ & $59(9.6)$ & & $18(11.5)$ & $9(25.7)$ & \\
\hline$\geq 2500-4000$ & $157(82.2)$ & $534(87.1)$ & & $131(83.9)$ & $26(74.2)$ & \\
\hline$\geq 4000$ & $7(3.7)$ & $20(3.2)$ & & $7(4.4)$ & 0 & \\
\hline Mode of delivery & & & 0.001 & & & 0.0001 \\
\hline Vaginal & $196(39.9)$ & $260(42.49)$ & & $135(33.9)$ & $61(65.6)$ & \\
\hline Cesarean & $295(60.1)$ & $353(57.6)$ & & $263(66.1)$ & $32(34.4)$ & \\
\hline Rank of children & & & 0.3 & & & 0.8 \\
\hline First child & $208(42.4)$ & $254(41.5)$ & & $170(42.7)$ & $38(41.3)$ & \\
\hline Second child and over & $282(57.6)$ & $358(58.5)$ & & $228(57.3)$ & $54(58.7)$ & \\
\hline \multicolumn{7}{|l|}{ Gestational age, wk } \\
\hline $35-37$ & $132(26.8)$ & $132(26.8)$ & & $120(30.2)$ & & \\
\hline $37-40$ & $347(70.9)$ & $347(70.9)$ & & $253(63.8)$ & & \\
\hline$>40$ & $11(2.3)$ & $11(2)$ & & $25(6.2)$ & & \\
\hline
\end{tabular}

\footnotetext{
$\mathrm{a}$ Data are presented as No.(\%).

b $\mathrm{N}=491$.

c $\mathrm{N}=398$.
} 
According to the study protocol, 491 (80\%) neonates revealed high TcB levels. This consisted of 240 (49\%) infants with bilirubin levels of $\geq 5 \mathrm{mg} / \mathrm{dL}$ during the first 24 hours and 251 (51\%) cases with bilirubin level $>8 \mathrm{mg} / \mathrm{dL}$ within 48 hours. TcB ranged $3.3-17.1 \mathrm{mg} / \mathrm{dL}$, average TcB level was1.7 $\pm 9.6 \mathrm{mg} / \mathrm{dL}$ (mean $6 \mathrm{mg} / \mathrm{dL}$ ) in first 24 hours and $2.1 \pm 9.1$ (mean $10 \mathrm{mg} / \mathrm{dL}$ ) in second 24 hours.

491 neonates with high TcB further underwent TsB examination using capillary method, of which 398 cases showed high TsB. Of the latter, 108 (27.1\%) cases showed bilirubin levels $\geq 5 \mathrm{mg} / \mathrm{dL}$ in first 24 hours and 290 (72.9\%) more than $8 \mathrm{mg} / \mathrm{dL}$ in second 24 hours (mean TsB $8.8 \mathrm{mg} / \mathrm{dL}$ ).

Based on these results, TcB has a positive predictive value (PPV) equal to $81 \%$ in the diagnosis of hyperbilirubinemia and the correlation coefficient between TcB and TsB is at most $72 \%$ (Pvalue $>0.001$ ).

$60.1 \%$ of infants in high TcB group and $66.1 \%$ of TsB positive group were delivered by Cesarean section ( $\mathrm{P}$ value $=$ 0.001). Based on this results, it seems that mode of delivery has a significant impact on bilirubin levels.

Other variables such as gender, weight, numerical, order and number of children did not show a significant impact on hyperbilirubinemia.

There was a $0.9 \% \mathrm{Rh}$ incompatibility (mother: Rh negative, baby: Rh positive) and a 13\% ABO incompatibility: mother O, baby A (7\%) and mother O, baby B (6\%).

358 neonates were infants of multiparous women. History of jaundice in previous deliveries of these mothers was reported in 60 (16.75\%) cases. Of these 42 (70\%) cases were treated by phototherapy and 18 (30\%) cases had not required any treatment. Among these 60 cases with previous history of jaundice 39 (65\%) cases were TsB positive and 21 (35\%) TsB negative.

In following days according to our pre-discharge advice, 40 (6.5\%) patients were readmitted for treatment. Mean bilirubin level in this group of infants was $12.2 \pm 4.5 \mathrm{mg} /$ $\mathrm{dL}$, and mean birth weight was $2671 \pm 426$ g. 13 (13.5\%) patients had $\mathrm{ABO}$ and 2 (5\%) Rh incompatibility. 38 newborns (95\%) were treated with phototherapy, only one case needed exchange transfusion.

\section{Discussion}

This study showed that TcB screening of hyperbilirubinemia in healthy term or late preterm neonates has highly predictive value and can be recommended for early detection of jaundice in nurseries. American academy of pediatrics (AAP) has also recommended this technique as a suitable and noninvasive method in assessment of hyperbilirubinemia progression (7).

In this study we assessed the correlation between TcB and TsB (as the gold standard test for bilirubin) in the diagnosis of jaundice with a PPV of $81 \%$. Several studies on the role of TcB as an inexpensive, suitable, and noninvasive tool for pre-discharge screening of jaundice in all neonates have been performed in different countries. In a study conducted in China on 113 neonates, TcB showed a good correlation with TsB (correlation coefficient: 0.83 and $P$ value $<0.001$ ). According to this study Bili check type 103 JM estimates bilirubin higher than serum levels (9), whereas in another study performed in Thailand with the same type of Bili check, correlation between TcB and TsB was reported as significant (correlation coefficient 0.8 and P-value < 0.001 ) and according to this study, this type of TcB reveals bilirubin levels about $0.7 \mathrm{mg} / \mathrm{dL}$ lower than serum levels (10). Also in our study. TcB and TsB were consistent (correlation coefficient 0.72 and $P$ value $<0.001$ ) confirming the results of two previous studies. It seems that Bili check type $8,000 \mathrm{KJ}$ with a PPV of $81 \%$ in the diagnosis of hyperbilirubinemia is a suitable tool for screening of neonatal jaundice.

In the study of Kaplan and colleagues, visual screening with TcB in identifying neonates with jaundice was made. They concluded that since blood sampling is not required in TcB, it is more suitable than TsB (11). In our study visual screening was not done.

Some studies revealed that routine pre-discharge TcB screening program is associated with significant reduction in the overall incidence of admission in neonates with higher bilirubin levels and phototherapy rate and reduced age at readmission for phototherapy and more frequent contacts with public health nurses after introduction of the TcB program (12). In our study $6.5 \%$ of patients had readmission for treatment.

High prevalence of hyperbilirubinemia with TcB and TsB measurements (about 80\%) in our study, was because of our low cut off levels obtained from Bhutani curve based solely on serum bilirubin levels. Normally the lowest pathologic level for TsB is $5 \mathrm{mg} / \mathrm{dL}$ in first 24 hours and $8 \mathrm{mg} / \mathrm{dL}$ in second 48 hours, whereas pathologic levels of bilirubin vary with some other factors such as gestational age, weight, and hours passed birth. As our study was the first expanded study of the kind performed in Iran on the prevalence of hyperbilirubinemia by means of TcB and TsB measurements and comparing, a precise cut off level was not available. In this regard more expanded studies with higher cut off levels need to be conducted. Recently in a publishment by institute of health economics about transcutaneous bilirubinometery for the screening of jaundice, is reported that pre-discharge TcB cut-off of $\geq 75$ th percentile at 48 to 72 hours is a good predictor of TsB of $\geq 95$ th percentile. If the cur-offs are set toward high sensitivity; a large number of false positives are acceptable and result in additional unnecessary TsB testing, but this is without serious clinical consequences. They emphasize that in TcB screening program, development of a local TcB nomogram, and selection of appropriate TcB cutoff is needed (13). Some researchers concluded that if TcB values were plotted on the TsB nomogram resulted in a trend towards a higher false negative rate but plotting TcB on transcutaneous nomogram resulted in better predictive value, with the best sensitivity (90.0\%) and specificity (87.79\%) (14), but we compared TcB and TsB measures simultaneously in the same nomogram. 
According to the present study, the prevalence of hyperbilirubinemia is higher in cesarean deliveries $(C / S)$ and in $\mathrm{ABO}$ and $\mathrm{Rh}$ incompatibility, which are similar to the results of other studies conducted in Thailand (9), China (8) and on Hispanic infants (15), although neonates delivered with $\mathrm{C} / \mathrm{S}$ stayed longer in nursery and detection of jaundice in these infants was more probable.

Estimation of bilirubin by TcB may be inaccurate in some situations, especially in severe cases of hyperbilirubinemia, in preterm infants and during phototherapy. We excluded preterm newborns from our study and used TcB just as a screening test. None of our cases received phototherapy, and because our study was done in a nursery where a high percentage of cases were discharged early from hospital, only a few of our patients had high bilirubin levels. we matched the results of TcB with our finding in physical examination at pre-discharge visits, it is logical that in icteric cases with underestimation of TcB test, we checked TsB to confirm the diagnosis of hyperbilirubinemia.

A limitation of our study was exclusion of the premature and low birth weight infants because precise diagnosis of hyperbilirubinemia in preterm infants is questionable and TcB measurements in this group of neonates should be further evaluated.

A point of strength in our study was more documented diagnosis of icterus with TcB in comparison with visual diagnosis in pre-discharge visits and more infants came back to recheck the bilirubin in follow up clinics, of which $6.5 \%$ were hospitalized to receive treatment. This was mainly (95\%) with phototherapy, and only in one case blood exchange was utilized. This highlights the importance of screening tests and parental notification towards the follow up of jaundice, especially in late preterms and low birth weight neonates.

TcB is an inexpensive, noninvasive and precise method for screening of hyperbilirubinemia with a high (81\%) PPV. According to high prevalence of neonatal jaundice, pre-discharge TcB measurement is recommended as the first line in screening of jaundice in all neonates.

\section{Acknowledgements}

We are thankful to neonatal health research center of Shahid Beheshti University of Medical Sciences for sup- porting the study and to nursery personnel of Mahdieh hospital.

\section{References}

1. Karon BS, Wickremasinghe AC, Lo SF, Saenger AK, Cook WJ. BiliChek transcutaneous bilirubin meter overestimates serum bilirubin as measured by the Doumas reference method. Clin Biochem. 2010;43(12):1009-12.

2. Hoppenot C, Emmett GA. Neonatal bilirubin triage with transcutaneous meters: when is a blood draw necessary? Hosp Pediatr. 2012;2(4):215-20.

3. National Association of Neonatal N. Prevention of acute bilirubin encephalopathy and kernicterus in newborns. Position Statement \#3049. NANN Board of Directors, March 2010. Adv Neonatal Care. 2010;10(3):112-8.

4. UK National Screening Committee. Neonatal screening for Kernicterus. UK NSC External Review. 2010.

5. el-Beshbishi SN, Shattuck KE, Mohammad AA, Petersen JR. Hyperbilirubinemia and transcutaneous bilirubinometry. Clin Chem. 2009;55(7):1280-7.

6. Newman TB. Universal bilirubin screening, guidelines, and evidence. Pediatrics. 2009;124(4):1199-202.

7. American Academy of Pediatrics Subcommittee on H. Management of hyperbilirubinemia in the newborn infant 35 or more weeks of gestation. Pediatrics. 2004;114(1):297-316.

8. Newman J. Re: Guidelines for detection, management and prevention of hyperbilirubinemia in term and late preterm newborn infants (35 or more weeks' gestation) - Summary. Paediatr Child Health 2007;12(5):401-7. Paediatr Child Health. 2007;12(7):613.

9. Lam TS, Tsui KL, Kam CW. Evaluation of a point-of-care transcutaneous bilirubinometer in Chinese neonates at an accident and emergency department. Hong Kong Med J. 2008;14(5):356-60.

10. Sanpavat S, Nuchprayoon I. Noninvasive transcutaneous bilirubin as a screening test to identify the need for serum bilirubin assessment. J Med Assoc Thai. 2004;87(10):1193-8.

11. Kaplan M, Shchors I, Algur N, Bromiker R, Schimmel MS, Hammerman C. Visual screening versus transcutaneous bilirubinometry for predischarge jaundice assessment. Acta Paediatr. 2008;97(6):759-63.

12. Wainer S, Parmar SM, Allegro D, Rabi Y, Lyon ME. Impact of a transcutaneous bilirubinometry program on resource utilization and severe hyperbilirubinemia. Pediatrics. 2012;129(1):77-86.

13. Mishra S, Chawla D, Agarwal R, Deorari AK, Paul VK, Bhutani VK. Transcutaneous bilirubinometry reduces the need for blood sampling in neonates with visible jaundice. Acta Paediatr 2009;98(12):1916-9.

14. Mohamed I, Blanchard AC, Delvin E, Cousineau J, Carceller A. Plotting transcutaneous bilirubin measurements on specific transcutaneous nomogram results in better prediction of significant hyperbilirubinemia in healthy term and near-term newborns: a pilot study. Neonatology. 2014;105(4):306-11.

15. Kolman KB, Mathieson KM, Frias C. A comparison of transcutaneous and total serum bilirubin in newborn Hispanic infants at 35 or more weeks of gestation. J Am Board Fam Med. 2007;20(3):266-71. 